\title{
Automatic Generation of Wrapper for Data Extraction from the Web
}

\author{
Suzhi Zhang ${ }^{1,2}$ and Zhengding $\mathrm{Lu}^{1}$ \\ College of Computer science and Technology, Huazhong University \\ of Science and technology, Wuhan, Hubei, China, 430074 \\ zhsuzhi@sohu.com \\ ${ }^{2}$ Department of Computer science and Technology, ZhengZhou Institute \\ of Light Industry, Zhengzhou, Henan, China, 450002 \\ zhsuzhi@sina.com
}

\begin{abstract}
With the development of the Internet, the Web has become invaluable information source. In order to use this information for more than human browsing, web pages in HTML must be converted into a format meaningful to software programs. Wrappers have been a useful technique to convert HTML documents into semantically meaningful XML files. In this paper, we propose a data extraction approach based on extracting schema, which generates automatically a wrapper to extract data from an HTML document, and produces an XML document conforming to given DTD. After the user defines extraction data schema in the form of DTD, the wrapper is generated automatically with the induction and leaning algorithm. The experiment indicates that the approach can correctly extract the required data from the source document with high accuracy.
\end{abstract}

\section{Introduction}

With the development of the Internet, the Web has become invaluable information source [1,2]. Enterprises publish information and goods price on the Web. To address the issues related to the effective use of information available on the Web, researchers from the database community view the Web as a large distributed database and apply database technologies to search information from the Web. However, HTML is a mark-up language originally designed for platform-independent formatting and displaying. Such Web pages are more suitable for human browsing, rather than consumption by computer programs[1]. How to extract the data on the Web into an integrated system is urgent work for many enterprise applications.

A popular approach to capture HTML information in a format meaningful to software programs is to write wrapper, which encapsulates the access to source and produces more structured data, such as XML[2]. However, developing and maintaining wrapper by hand turn out to be slow, labor-intensive and error prone[2]. In this paper, we propose a data extraction approach based on defined schema that can generates automatically a wrapper to extract data from HTML document, and produces an XML document conforming to given DTD. 


\section{System Architecture}

The process of wrapper generation is implemented by a software tool, which takes a URL as input and output is a set of mapping rules between information Blocks of Interest (BOI) on the Web and user defined schema. A wrapper is essentially a set of such rules. Figure 1 shows the system architecture of the software tool, called Awdgs, that is automatic Web data extraction guided by schema. It can convert the HTML document into meaningful XML data conforming to defined schema.

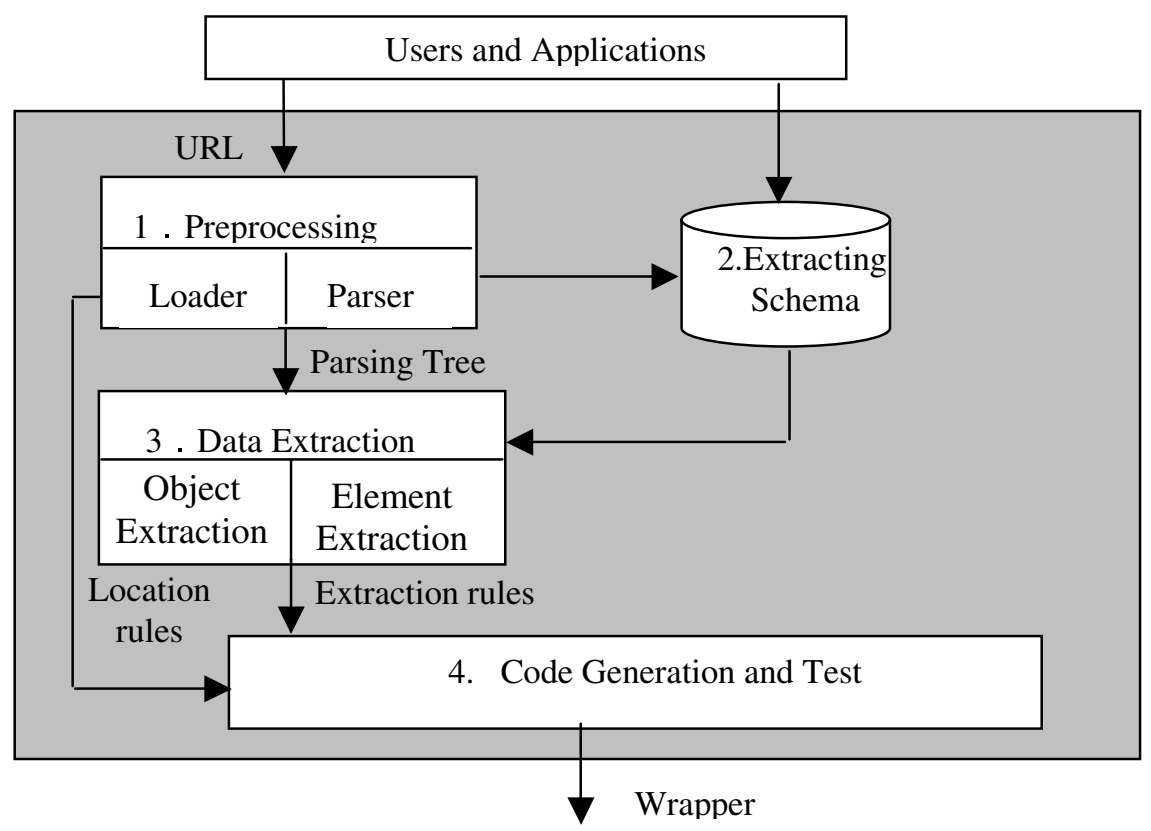

Fig. 1. System Architecture

Preprocessing includes two steps: loader and parser. Loader fetches the web page using the URL given by the user. The fetched HTML source is parsed into an HTML tree. Extraction Schema is defined by user based the parsing tree and interested data on the Web page. Data Extraction is divided into two steps, (1) Object extraction identifies interested regions in the Web pages, and Element extraction identifies the elements inside of the objects. A data object typically consists of several elements that are separated by a group of element separators., such as $<\mathrm{td}>$ in an HTML table. Code Generation and Test generates the wrapper program code through extraction roles. The rules of extracting data define the mapping between HTML document structure and defined schema. By such a set of mapping rules, the extracted data can be located in the HTML document. 


\section{System Realization}

\subsection{Fetching Web Document and Tree Representation}

The HTML document is fetched by its URL, and the given page is transformed into a well-formed document. A well-formed document defined in [2]. It can be modeled as a tag tree.

Define[Minimal Subtree]: Let $\mathrm{T}=(\mathrm{V}, \mathrm{E})$ be a tag tree of a web document $\mathrm{D}$ where $\mathrm{V}=\mathrm{V}_{\mathrm{T}} \cup \mathrm{V}_{\mathrm{C}}, \mathrm{V}_{\mathrm{T}}$ is a finite set of tag node (internal nodes) and $\mathrm{V}_{\mathrm{C}}$ is a finite set of content nodes(leaf nodes); $\mathrm{E} \subset(\mathrm{Vx} \mathrm{V})$, representing the directed edges. A minimal subtree with property $\mathrm{p}$ is a subtree anchored at node $\mathrm{u}, \mathrm{u} \in \mathrm{V}$, and there is no other subtree $\mathrm{w}, \mathrm{w} \in \mathrm{V}$, which satisfies both the property $\mathrm{P}$ and the condition that $\mathrm{u}$ is an ancestor of $w$.

\subsection{Object Location and Extraction}

By visual choosing interested data object in HTML tree, the minimal subtree and its location in the HTML tree are generated. We adopt three methods, Largest Tag Count, Highest Fanout and Largest Size Increase, and some heuristic methods to get the minimal subtree, which are similar to [2]. It can achieve higher accuracy.

\subsection{Extracting Schema}

An HTML tree represents the syntactic structure of an HTML document. It does not reflect the semantics of the data [2]. When extracting data in integrated system, we should know which data are our needed. For example, Figure 2 is defined schema for data extraction from object subtree, and its corresponding schema tree.

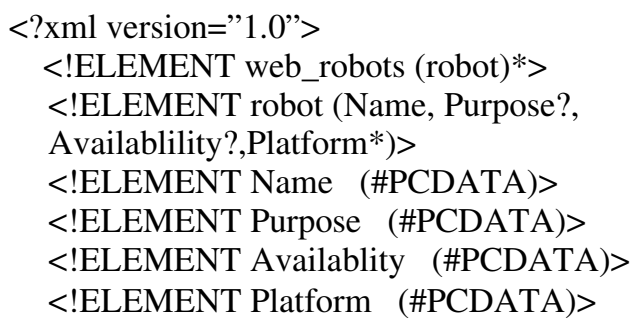

\section{$<x$}

$<$ !ELEMENT web_robots (robot)*> $<$ !ELEMENT robot (Name, Purpose?, Availablility?,Platform*)> $<$ !ELEMENT Purpose (\#PCDATA) > $<$ !ELEMENT Platform (\#PCDATA) $>$

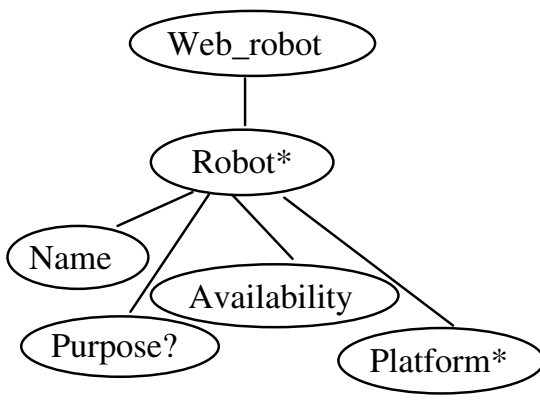

Fig. 2. Extracting Schema in DTD and Schema Tree 
The corresponding extraction rules as follows:

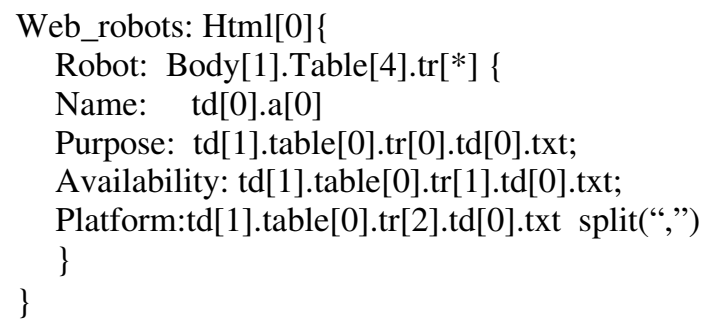

\subsection{Rule Extraction Algorithm}

Rule extraction algorithm purposed in this paper belongs to induction learning method. The main process of the wrapper generation consists of two steps: (1) obtaining initial mapping rules; (2) adopting induction learning algorithm in order to get extracting rules.

Foe example, the mapping rule instance is

Web_robots.Robot.Name=Html[0].Body[1].Table[4].table[0].tr[1].td[0].a[0]

When extracting data from HTML document into XML, the element of Name in the XML document can be generated by the rule, formatted as following $<$ Name $>$ Acme.Spider $<$ /Name $>$

The induction learning algorithm is based on obtaining initial mapping rules and extracting schema. The algorithm takes a list of initial mapping rules $\mathrm{L}$ as input and returns automatically an extraction rules as output for the web page.

\section{Experimental Results and Conclusions}

The objective of usability test is to evaluate how easy it is to use the system and whether the algorithm is effective. In the experiment, we take three web pages, including Quotes, Web robots and Amazon books, as the test subjects. We use the time-cost by the user and the system as an indicator to show how easy the system can be used. Experimental data are omitted for the limited space reason, but the results indicate the algorithm is effective and correct.

A lot of technologies and systems have been proposed about web data wrapper in recent year, such as WIEN[6], Muslea[4], TSIMMIS[3], XWRAP[1], ARIADEN[5] etc. Our contributions in the paper are (1) With the guidance of extracting schema, the generated wrapper can be more accurate and better reflect user requirements. (2) The generated XML document conforms to the defined schema. (3) Mapping rules will be generated automatically with induction learning Algorithm.

\section{References}

1. L. Liu, C. Pu, W. Han. XWRAP: An XML-enabled wrapper construction system for web information sources. In Proc. The International Conference on Data Engineering (ICDE), San Diego,California,USA,2000 
2. W. Han, D. Buttler, C. Pu. Wrapping Web data into XML. SIGMOD Record, Vol. 30, No.3, September 2001.

3. J.Hammer, M. Brenning, H. Garcia-Molina et al. Template-based wrappers in the TSIMMIS system. In Proc. ACM SIGMOD’97. May,1997.

4. I. Muslea, S. Minton, C. Knoblock. A hierarchical approach to wrapper induction. In Proc. The 3rd International Conference on Autonomous Agents (Agents'99). Seattle,WA, USA, 1999.

5. C. A. Knoblock, S. Minton, J. L. Ambite et al. Modeling web source for information integration. In Proc. AAAI'98, Madison, WI, 1998.

6. N. Kushmerick, D. Weil, R. Doorenbos. Wrapper induction for information extraction. In proc. Int. Joint Conference on Artificial Intelligence (IJCAI'97), Nagoya, Japan,1997. 